\title{
Development and Implementation of a Surveillance Network System for Emerging Infectious Diseases in the Caribbean (ARICABA)
}

\author{
WonGyu Lewis Kim ${ }^{1,2}$, Chelsea Anne Ducharme ${ }^{2}$, Bernard Jean-Marie Philippe Bucher ${ }^{3,4}$ \\ ${ }^{1}$ University of Illinois at Chicago, ${ }^{2}$ School of Public Health and Health Service, The George \\ Washington University, ${ }^{3}$ Centre International Pour La Coopération Médicale et Sanitaire \\ dans la Caribbean et les Amériques, ${ }^{4}$ University College London
}

\section{Abstract}

Dengue fever, including dengue hemorrhagic fever, has become a re-emerging public health threat in the Caribbean in the absence of a comprehensive regional surveillance system. In this deficiency, a project entitled ARICABA, strives to implement a pilot surveillance system across three islands: Martinique, St. Lucia, and Dominica. The aim of this project is to establish a network for epidemiological surveillance of infectious diseases, utilizing information and communication technology. This paper describes the system design and development strategies of a "network of networks" surveillance system for infectious diseases in the Caribbean. Also described are benefits, challenges, and limitations of this approach across the three island nations identified through direct observation, open-ended interviews, and email communications with an on-site IT consultant, key informants, and the project director. Identified core systems design of the ARICABA data warehouse include a disease monitoring system and a syndromic surveillance system. Three components comprise the development strategy: the data warehouse server, the geographical information system, and forecasting algorithms; these are recognized technical priorities of the surveillance system. A main benefit of the ARICABA surveillance system is improving responsiveness and representativeness of existing health systems through automated data collection, process, and transmission of information from various sources. Challenges include overcoming technology gaps between countries; real-time data collection points; multiple language support; and "componentoriented" development approaches.

Keywords: outbreak, surveillance, syndromic surveillance, forecasting, emerging infectious diseases, Caribbean, 


\title{
Development and Implementation of a Surveillance Network System for
}

Emerging Infectious Diseases in the Caribbean (ARICABA)

\section{Introduction}

In 1999, the Association of Caribbean States (ACS) recognized emerging infectious diseases as a major challenge after a large dengue fever epidemic in Cuba and made a call for proposals for a comprehensive regional surveillance system. In 2005, a preliminary study entitled Vigilia, was conducted from Martinique to test strategies, models, and hypotheses for integrated surveillance in the Caribbean. The pilot system demonstrated early warning capacity based on inference from collected data by correctly predicting an upcoming outbreak. Martinique's Regional Council and the Centre International pour la Coopération Médicale et Sanitaire dans la Caribbean et les Amériques (CICOMSCA) initiated a project entitled ARICABA, meaning "to look" in the Carib language. ARICABA incorporates lessons learned from Vigilia to implement a surveillance system across three islands: Martinique, St. Lucia, and Dominica. This project has been funded by the European Union with an approximately $€ 3$ million total budget (6).

\begin{abstract}
About ARICABA
ARICABA, with cooperation of the Regional Council of Martinique, St. Lucia, and Dominica governments, envisions a comprehensive emerging infectious disease surveillance system to detect and forecast threats and ultimately protect the citizens and visitors to the three participating islands. The mission of ARICABA is to heighten protection of Caribbean residents and visitors by closing the gap in existing infectious disease surveillance systems, especially emerging threats such as dengue fever and influenza. The combination of global and local partnerships; systems strengthening and integration; and the development of mathematical modeling will permit improved detection and forecasting of selected diseases. To accomplish this mission, the aim is to establish a network for epidemiological surveillance of infectious diseases, based on information collected through various sources of data.
\end{abstract}

\section{Country Background Information}

It is essential to contextualize the countries in which the system will function prior to describing surveillance system design. Understanding Martinique, Dominica and St. Lucia is imperative to designing a surveillance system suitable for their social, economic, and political environments. Table 1 illustrates background country information including health indicators and health systems (6).

\section{Martinique}

Martinique, colonized by France in 1635, remains one of several French overseas departments in the Caribbean (4). It measures a total of 1,060 square $\mathrm{km}$; the island is home to a mountainous terrain, tropical climate, and a five-month rainy season (4). The population is approximately 403,688 and inhabitants speak mainly Creole and French (4). According to recent data, the GDP (PPP) was $\$ 4.5$ billion. Martinique is relatively more "westernized" and developed as compared to the other pilot countries (5).

\section{Dominica}

Dominica, located directly north of Martinique, has a population of 72, 969 persons (4). Presently an independent country, this 751 square km island is known as the "nature island" due to its lush, varied flora and rugged volcanic terrain. English is the official language though French patois, a form of Creole, is widely spoken (4). Dominica has the lowest income among the three pilot countries. Politically, the Dominican Ministry of Health is very well respected, including their 
Epidemiology Unit. Their Health Information Technology Unit is housed under the Epidemiology Unit which eases coordination among the two teams.

\section{St. Lucia}

Though possession transferred between Britain and France fourteen times, St. Lucia was granted independence in 1979 by England (4). Bordering Martinique to the south, approximately 161, 557 persons inhabit this tropical 606 square $\mathrm{km}$ island (4). English is the official language of this island (4). As an independent country, there exists a Ministry of Health, an Epidemiology Unit and a Health Information Unit. St. Lucia is currently developing its own surveillance system: SLUHiS.

Table 1. Country Information

\begin{tabular}{|c|c|c|c|}
\hline & Dominica & Martinique & St. Lucia \\
\hline \multicolumn{4}{|l|}{ Basic Information } \\
\hline Size & 751 square $\mathrm{km}$ & 1,060 square $\mathrm{km}$ & 616 square $\mathrm{km}$ \\
\hline Population & 72,660 & 436,131 & 173,765 \\
\hline Political Status & $\begin{array}{l}\text { Parliamentary Republic, } \\
\text { independent since } 1978\end{array}$ & $\begin{array}{l}\text { Overseas Region of } \\
\text { France, part of the } \\
\text { European Union }\end{array}$ & $\begin{array}{l}\text { Parliamentary } \\
\text { Democracy, } \\
\text { independent since } \\
1979\end{array}$ \\
\hline $\begin{array}{l}\text { GDP } \\
\text { (Purchasing Power } \\
\text { Parity) }\end{array}$ & $\$ 380$ million & $\$ 4.5$ billion & $\$ 1.75$ billion \\
\hline \multicolumn{4}{|l|}{ Health Indicators } \\
\hline Life Expectancy & $\begin{array}{l}\text { Male: } 72 \text { years } \\
\text { Female: } 76 \text { years }\end{array}$ & $\begin{array}{ll}\text { Male: } 79 & \text { years } \\
\text { Female: } 78 \text { years } & \end{array}$ & $\begin{array}{l}\text { Male: } 72 \text { years } \\
\text { Female: } 78 \text { years }\end{array}$ \\
\hline Fertility Rate & 2.08 children born/woman & $\begin{array}{ll}1.79 & \text { children } \\
\text { born/woman }\end{array}$ & $\begin{array}{l}1.82 \text { children } \\
\text { born/woman }\end{array}$ \\
\hline $\begin{array}{llr}\text { Probability } & \text { of } \\
\text { dying } & \text { under } & \text { five } \\
\text { (per } 1,000 & \text { live } \\
\text { births) } & & \\
\end{array}$ & 15 & 7.44 & 14 \\
\hline $\begin{array}{l}\text { \% years lost due to } \\
\text { communicable } \\
\text { diseases }\end{array}$ & $20 \%$ & (not available) & $17 \%$ \\
\hline $\begin{array}{l}\text { Existing Health } \\
\text { Surveillance }\end{array}$ & $\begin{array}{l}\text { Health Information Unit, } \\
\text { CAREC, } \\
\text { National Public Health } \\
\text { Surveillance and Response } \\
\text { Team, National Health } \\
\text { Information System in } \\
\text { development }\end{array}$ & $\begin{array}{l}\text { Health Unit in General } \\
\text { Council of Martinique, } \\
\text { Regional Council of } \\
\text { Martinique (health } \\
\text { planning) }\end{array}$ & $\begin{array}{l}\text { Epidemiology } \\
\text { Unit, CAREC, } \\
\text { National Health } \\
\text { Information } \\
\text { System in } \\
\text { development }\end{array}$ \\
\hline
\end{tabular}




\section{Research methods}

ARICABA project data was mainly collected through three three-month fellowships through the Global Health Service Fellow Scholarship program at the Center for Global Health at the George Washington University from September 2010 to August 2011. Data collection occurred in Martinique, St. Lucia and Dominica, where the ARICABA pilot project functions.

Data collection techniques include meetings, direct observation, open ended interviews and email communications with an in-site IT consultant, key informants and project director, and direct observation; this data was shared openly among all fellows. Information was also obtained through review of ARICABA technical documents, literature reviews and web searches. Key search words included surveillance system, syndromic, and outbreak detection. Web search engines utilized include PubMed, MD Consult, Science, and Google.

Before surveillance system design, target infectious diseases were identified; dengue fever was chosen as a model for a vector-borne disease while influenza-like illnesses were chosen to represent man-to-man transmission (2). Dengue fever and influenza-like illnesses were chosen for surveillance in this project based on prevalence, modes of transmission, and complexity (2).

As a network of networks, the ARICABA surveillance system ideally consists of linked databases of the three countries that connect to all public and private hospitals, public and private labs, community health center, pharmacies, airports and seaports. Figure 1 shows that each country's health system will link to its data collection points (DCPs) and the DCPs will link to a data warehouse where the automated early detection analyses will operate. In this paper, the design of the surveillance system will focus on the data warehouse rather than DCPs since each DCP will be unique to the country's IT infrastructures and political structure (ex: health units). Nonetheless, all stakeholders will provide necessary data that allows data analyses within the warehouse based on an agreed upon ARICABA Charter.

The design of system of the data warehouse as well as data collection should ideally:

- Monitor confirmed disease cases;

- Forecast upcoming outbreaks;

- Capture necessary data from DCPs such as symptoms, meteorological conditions, overthe-counter (OTC) sales, human migration, mosquito distribution, etc; and

- Effectively disseminate data and information.

Likewise, the development strategies should meet the needs of:

- Size and the Operation System (OS) of the ARICABA Data Warehouse;

- Information dissemination tools; and

- Forecasting algorithms. 


\section{Results}

\section{Design}

There are two components in the data warehouse. The first is a conventional disease monitoring system that collects suspected, probable, and confirmed cases; type of disease; and date of event and report. The other component is a syndromic surveillance system that collects syndrome of diseases from data contributors that allows automated epidemic detection for early warning systems. The rationale of having two components in one system is to maximize the system's "software ergonomics" to match with the public health professional's work flow (9). Since public health professionals tend to spend more time with disease monitoring and intervention implementation rather than detecting the outbreak itself, the system should be able to detect the outbreak and also enable the ability to monitor the disease and progress of interventions spatially and temporally. Therefore, the data warehouse will receive all data from the DCPs. Then, the syndromic system will function to detect the outbreak and the disease monitoring system will demonstrate the location and time of the outbreaks and allow evaluation of interventions.

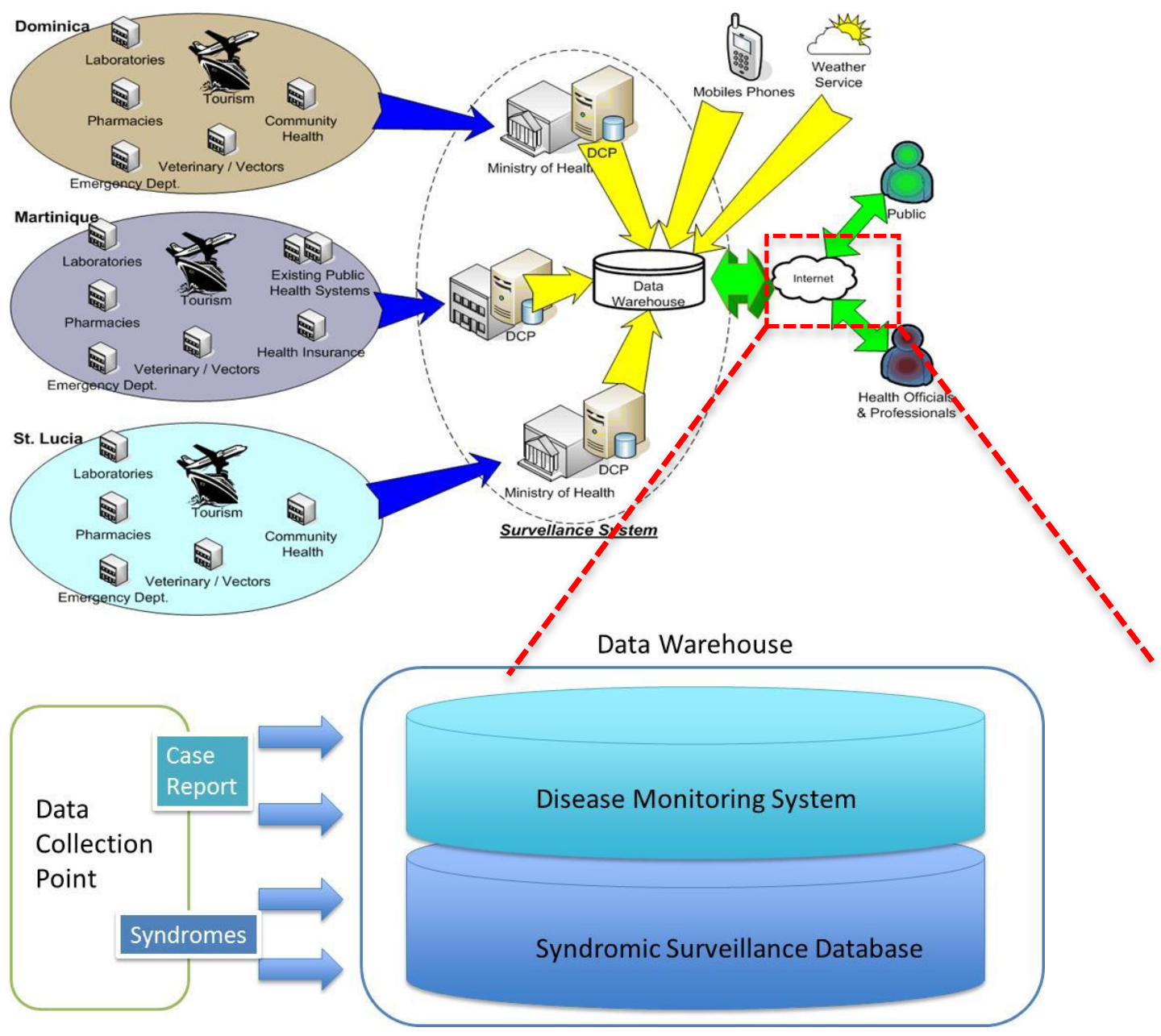

Figure 1. Network of Networks Overview 


\section{Development and Implementation of a Surveillance Network System for}

Emerging Infectious Diseases in the Caribbean (ARICABA)

\section{1) Disease monitoring system}

As previously mentioned, the disease monitoring system will collect suspected, probable, and confirmed cases based on a case definition from health providers and laboratories to track the diseases retrospectively. This section will discuss case definition of dengue fever, dengue hemorrhagic fever, and influenza-like illness as well as data sources, contents, and dissemination method of the disease monitoring system.

i) Develop case definition

a. Dengue fever (2):

i. Suspected

Defined by the association of at least:

- Sudden-onset high-grade fever $\left(\geq 38.5^{\circ} \mathrm{C}\right)$ of less than 10 days duration,

- Pain: headache \pm joint pain \pm muscle pain \pm back pain, and lack of any infectious focus.

ii. Probable

Either a case of suspected Dengue with at least two of the following clinical and biological criteria:

- Skin rash

- Minor signs of bleeding

- Thrombocytopenia (platelets $<100,000 / \mathrm{mm} 3$ )

- $\mathrm{CRP}<30 \mathrm{mg} / \mathrm{l}$ or a suspected case of Dengue occurring during an outbreak.

iii. Confirmed

A suspected or probable case of Dengue confirmed by at least one of the following laboratory tests:

- MAC-ELISA of a single serum sample evidencing specific IgM,

- Serum culture or PCR identifying the Dengue virus, significant rise in specific

b. Dengue haemorrhagic fever (2): Fever with at least one haemorrhagic (bleeding) manifestations - i.e. purpura, epistaxis, hemoptysis, and melena -- with or without jaundice.

c. Influenza like illness (2):

i. Suspected

- Undifferentiated Fever

- Fever and Respiratory symptoms

ii) Determine data source (type of system)

The scope of data sources should focus on where, who, and when: geographic area, populations and time period to be covered. Additionally, the scope must define how much data must be collected.

In Martinique, data sources are public and private hospitals, including the University Hospital in Fort de France, the largest hospital in Martinique. Private and public laboratories are also key 


\section{Development and Implementation of a Surveillance Network System for Emerging Infectious Diseases in the Caribbean (ARICABA)}

data sources. Data should be collected on a weekly basis or a request basis, dependent on data. The amount of data should fulfill the list of data contents.

In St. Lucia and Dominica, the main data source will be epidemiology unit databases in the Department of Health for the reported cases. Laboratories, public, and private hospitals are also data sources dependent on IT infrastructure. Data should be collected on a weekly basis or a request basis, dependent on data. The amount of data should fulfill the list of data contents.

iii) Determine data contents

From the data source, specific information about each individual, health care provider, or event should be collected. Data contents for the disease monitoring system should describe the context of an outbreak including where, when, and to whom it occurred. The contents should be able to show types, sources, and treatment of diseases to assist in determining effective interventions. The data contents to be collected from data sources are date reported; disease; subtype; county; location name; date of event; latitude; longitude; diagnosis source; disease description; diagnosis date; dates of treatment; and date of death (see Appendix I).

Ideally, data should be collected in real-time. However, every eight hours or daily reporting is acceptable. In addition, the data should be collected on a request basis.

iv) Develop data-collection instruments

The resource to gather the data contents from the data sources should be identified.

In Martinique, the main instruments to collect the data will be computerized information systems such as email and web sites since IT systems are available in health facilities. However, phone and fax can also be used.

Due to challenges in information systems and technology in low-resource settings such as St. Lucia and Dominica, phone and fax are currently important data collection instruments for hospitals, laboratories, and community health centers. Computerized information systems should be implemented to enhance data collection speed and accuracy.

v) Develop and test analytic approach

The collected data should be processed and analyzed. The data entry process will be separate from data coding in order to minimize data transcription or coding errors (9). During data entry, the staff member must check and edit data before entering into the data warehouse.

Routine analyses will be used for conditions of target diseases. Baseline data will include five years of backlog reporting history in Martinique, St. Lucia and Dominica. National data will be utilized for the unit of analysis, presenting time, person, and place. It must show whether or not the diseases have decreased or increased as a ratio in a weekly period (9). Updated or corrected data will be used for later reports and analyses. A sensitivity and predictive value positive of the method must be presented. 


\section{Development and Implementation of a Surveillance Network System for}

Emerging Infectious Diseases in the Caribbean (ARICABA)

\section{vi) Develop dissemination mechanism}

A data dissemination mechanism will focus on ensuring that those who need the information will receive it (9). After analyzing the data, the findings should be disseminated to public health officials and the public, if applicable. The findings from the disease monitoring system should be written as a report and must contain the number of hospitalized, number of deaths, and number of confirmed cases, including serotype. The dissemination mechanism for the data will be mainly web-based. Public health officials or professionals will log into a secure web site to update the analyzed data and post a report. Email is also suitable to send a report for key informants. Important findings also should be available to the public through a website and computerized social networks such as Facebook and Twitter.

vii) Ensure use of analysis and interpretation

It is important to evaluate the usefulness of data that is collected to those who use it (9). The evaluation will be conducted by a monthly survey to public health officials and professionals. Also, web-statistics will be used to see how many people access which information and for how long. The survey will also include what other information would be useful in future analyses.

\section{2) Syndromic Surveillance System}

The syndromic surveillance component will enable outbreak detection at early stages. This section will describe data sources and collection strategies; data analysis and outbreak detection methods; and data visualization, information dissemination and reporting of the ARICABA syndromic surveillance system. The design of the syndromic surveillance system focuses on providing flexible and scalable infectious disease information, sharing (across species and jurisdictions), alerting, analyses, and visualization platforms $(7,10)$. Also, the system needs to support interactive, dynamic, spatial-temporal analysis of epidemiological textual and sequential data.

\section{i) Data sources and collection strategies}

For the syndromic surveillance system, timely data should be provided with electronic prediagnosis health indicators (10). Data sources for this system will include healthcare providers, schools, pharmacies, laboratories, and military medical facilities. From the source, data such as chief complaints from emergency department visits; ambulatory visit records; hospital admissions; OTC drug sales from pharmacy stores; triage nurse calls; emergency calls; work or school absenteeism data; veterinary health records; laboratory test orders; and health department requests for influenza testing will be collected to monitor syndromes. It will also collect demographic data such as gender, age, area of residence and data relevant to patient visits. The syndromes to be monitored in this system include rash, fever, respiratory distress, hemorrhagic illness, severe illness, and death. 
Table 2. Data collection and sources for the data warehouse

\begin{tabular}{|c|c|c|}
\hline & Disease Monitoring Database & $\begin{array}{l}\text { Syndromic } \quad \text { Surveillance } \\
\text { Database }\end{array}$ \\
\hline Collect data & $\begin{array}{l}\text { Date Reported } \\
\text { Disease } \\
\text { Subtype } \\
\text { County } \\
\text { Location Name } \\
\text { Date of Event } \\
\text { Latitude } \\
\text { Longitude } \\
\text { Diagnosis source } \\
\text { Disease description } \\
\text { Diagnosis date } \\
\text { Dates of treatment } \\
\text { Date of death }\end{array}$ & $\begin{array}{l}\text { Emergency department visit chief } \\
\text { complaints, } \\
\text { Ambulatory visit records, } \\
\text { Hospital admissions, } \\
\text { Over the counter (OTC) drug sales } \\
\text { from Pharmacy stores, } \\
\text { Triage nurse calls, } \\
\text { Emergency calls, } \\
\text { Work or school absenteeism data, } \\
\text { Veterinary health records, } \\
\text { Laboratory test orders and results, } \\
\text { Health department request for } \\
\text { influenza testing }\end{array}$ \\
\hline Data sources & $\begin{array}{l}\text { Healthcare providers } \\
\text { Laboratories }\end{array}$ & $\begin{array}{l}\text { Healthcare providers } \\
\text { Schools } \\
\text { Pharmacies } \\
\text { Laboratories } \\
\text { Military medical facilities. }\end{array}$ \\
\hline Monitoring & $\begin{array}{l}\text { Suspected cases } \\
\text { Confirmed cases (including } \\
\text { types) } \\
\text { Number of hospitalized } \\
\text { Number of death }\end{array}$ & $\begin{array}{l}\text { Rash } \\
\text { Fever } \\
\text { Respiratory } \\
\text { Hemorrhagic illness } \\
\text { Severe illness and death }\end{array}$ \\
\hline
\end{tabular}

Based on the data collection and source identified, Figure 3 shows main entities and attributes of the ARICABA syndromic surveillance system in the database. 
Development and Implementation of a Surveillance Network System for

Emerging Infectious Diseases in the Caribbean (ARICABA)

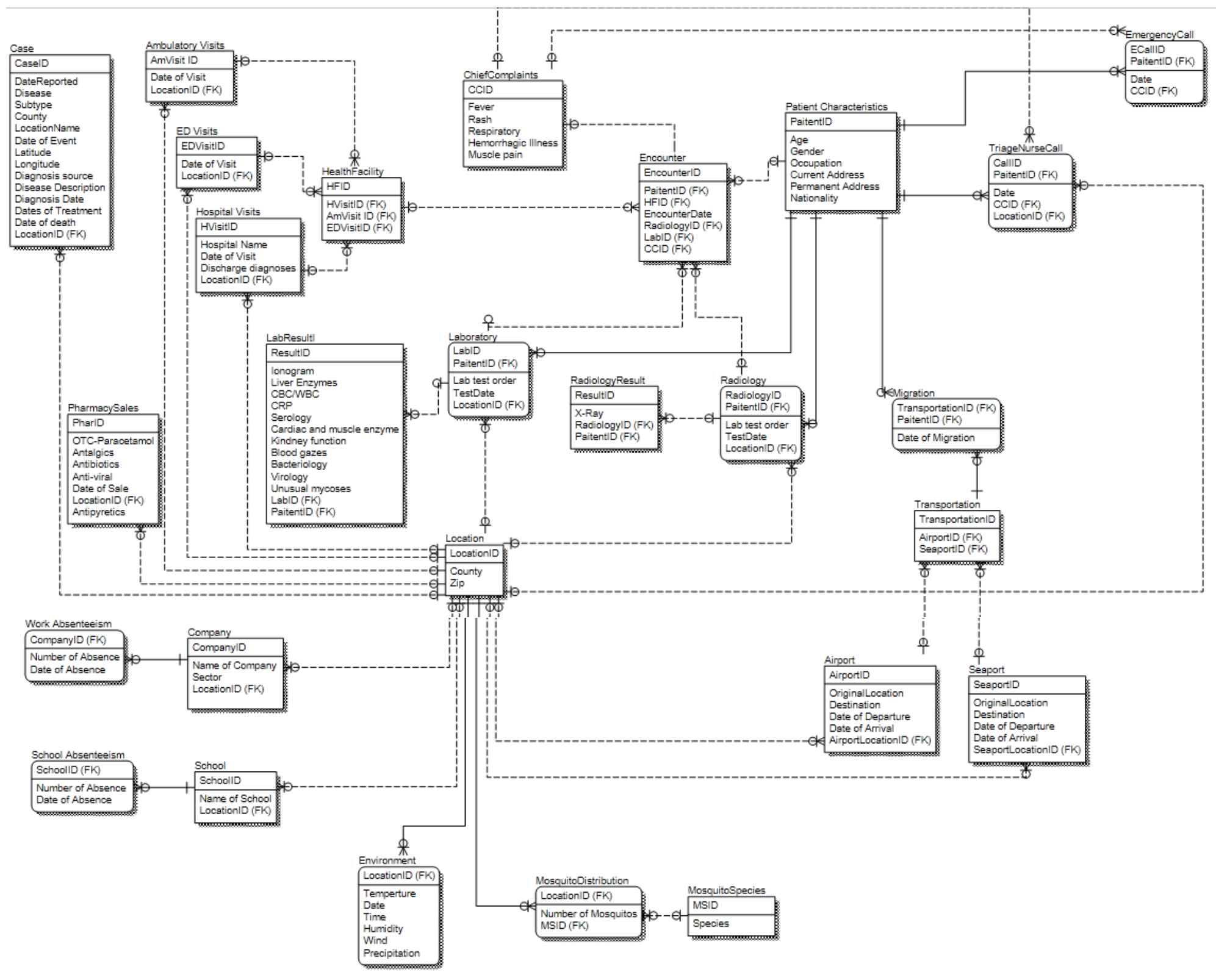

Figure 2. ARICABA ERD (Entity Relationship Diagram)

For the collection strategy, these data will be collected in real-time through HL7 messages from other computer systems such as registration systems and laboratory information systems, over a Secure Shell-protected Internet connection in an automated mode. For effectiveness and validity of data usage for illness pattern detection, it is important to consider a possible time lead compared with diagnosis (10). For example, it should compare chief complaints and discharge diagnosis to cross check from multiple sources to ensure similarity. 
ii) Data analysis and outbreak detection

In order to analyze collected data for outbreaks, the first step is to define syndrome classifications (3). It will apply a keyword classifier and ICD-10 classifier to chief compliant data for a syndrome classification. As a syndrome classifier, the Chief Complaint Coder (CoCo) module, which is based on Bayesian classifiers and has been used in The Real-time Outbreak and Disease Surveillance (RODS) system in the University of Pittsburgh, will be utilized. The Co-Co module has been proven effective through the RODS system. Since the size of the geographical tested area of the RODS system is similar to the ARICABA project, the Co-Co module may also be an effective syndrome classifier for the ARICABA project. Therefore, data analysis and outbreak detection algorithms of RODS can be modified and applied to ARICABA's data analysis and outbreak detection algorithms.

iii) Data visualization, information dissemination, and reporting

Like the disease monitoring system, it is important to make available a sophisticated spatialtemporal visualization environment to help visualize public health case reports and analyze results in both retrospective and prospective spatial-temporal clustering approaches (Zeng 2008). The ARICABA syndromic surveillance system will provide multiple graphing techniques with both time-series and geographical displays available via password-protected Web interface. It must be able to provide the following (10):

- Time-series plots updated on each syndrome daily. The user will be able to view these graphs by county or for the three countries.

- An interactive view categorized by the syndrome, region, start dates, and end dates, to generate customized time-series plots.

- Location mapping by use of geographic information system (GIS), which can display disease cases' spatial distribution using patients' zip code information.

\section{Development Strategies}

This paper will focus on development of the software and exclude hardware requirements. The hardware requirements will be determined based on the volume of data from the DCPs and the Internet connection capacity. Thus, we will discuss the software for the server such as operating system (OS), database, web interface, programs for data analysis, and the geographical information system (GIS) for data visualization.

\section{Data warehouse}

Data warehouses will be implemented in Martinique because it has the most advanced IT infrastructure among the three countries. The data warehouse will be linked to DCPs to receive the collected data (Figure 3). The collected data will go through an extract, transform and load (ETL) process for data cleaning (Figure 3). 
Microsoft Windows Server 2008 will be used as the OS. The rationale for selecting a Windows Server system instead of a Linux or Sun system is due to ease of IT technician recruitment for Windows system in Martinique; it is important to consider local IT human resources for creation of IT systems in developing countries.

ER Win 4.0 is used to develop the Entity Relationship Diagram (ERD), which allows development of a logical and a physical model of data modeling for the data warehouse. The ERD is developed based on a platform of Microsoft SQL Server. The data warehouse will also have a data mart for data analysis through mathematical algorithms.

The server will provide Internet Information Service (IIS) Web service for a web site. The web interface will be developed with ASP.NET language in order to display data, provide interactive user-interface via the website.

The data warehouse will also provide a GIS application server component with ArcIMS (Figure 3). These web and GIS servers will be implemented in a separate server machine in order to lessen the burden of the data warehouse.

a. Disease monitoring system

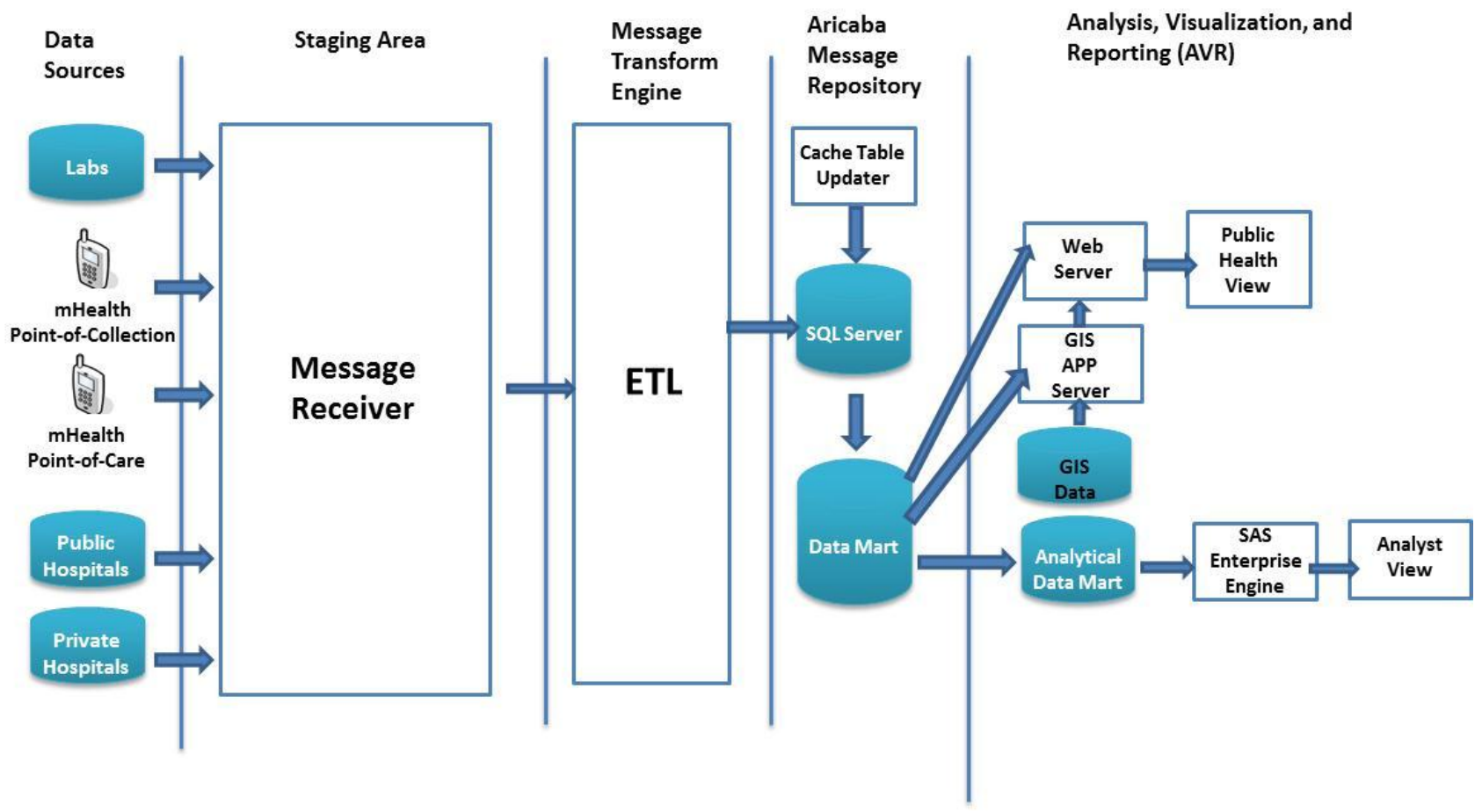

Figure 3. Data Warehouse Architecture 
Development and Implementation of a Surveillance Network System for

Emerging Infectious Diseases in the Caribbean (ARICABA)

b. Syndromic surveillance system

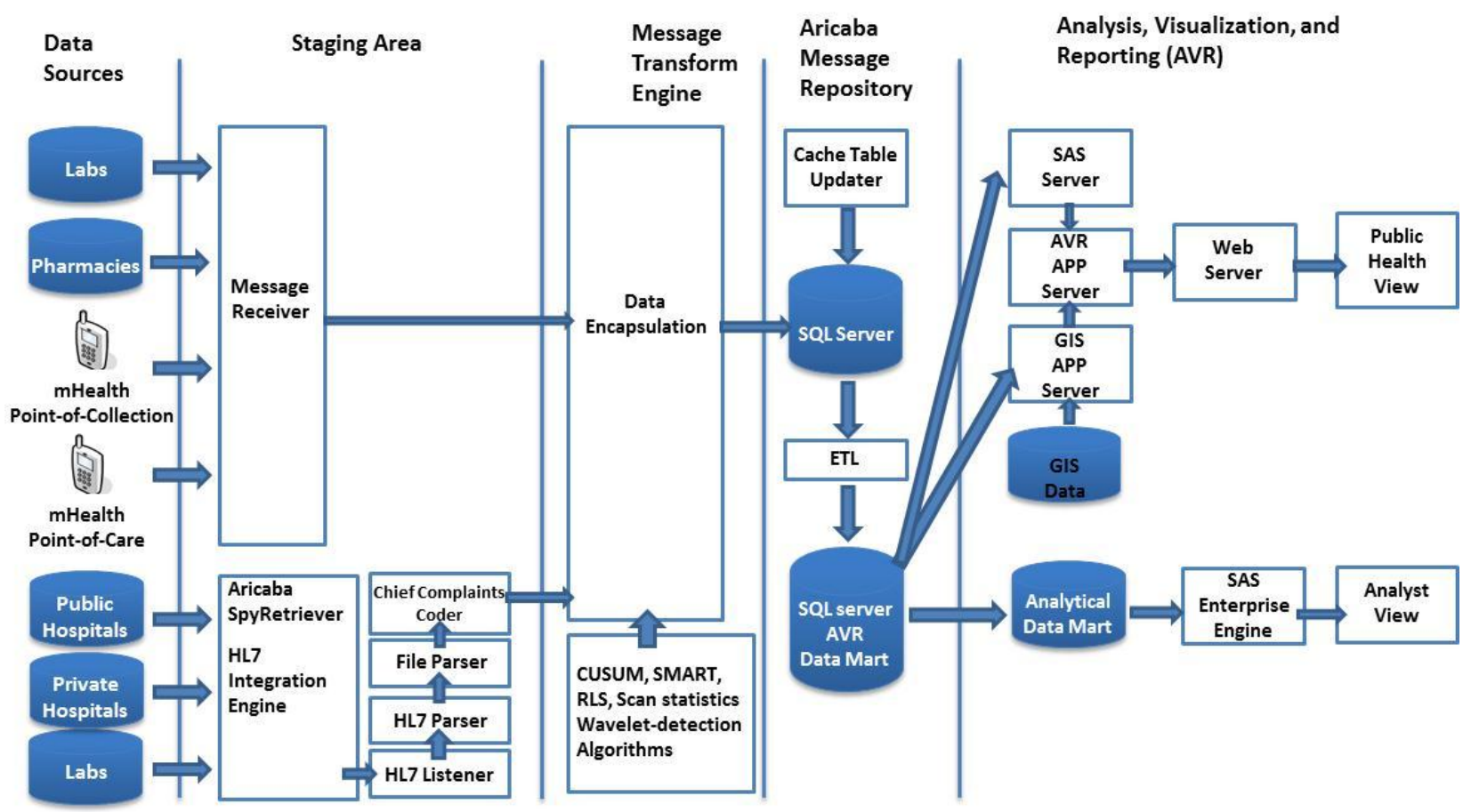

Geographical Information System

GIS will be developed to demonstrate mappings of the incidence and prevalence of diseases and health facilities for interventions. The ArcGIS 9.3 version will be used as the GIS system.

The mapping of incidence and prevalence of diseases will be developed for all three countries: Martinique, St. Lucia, and Dominica. Therefore, incidence and prevalence of disease data must include location, including longitude and latitude.

Health facility mapping must also be developed among the three countries. Health facilities include public and private hospitals, clinics, and laboratories. For more interactive maps, ArcGIS API for Flex will be used to create a flash file for the web.

\section{Forecasting algorithm}

For the outbreak detection algorithms, multiple forecasters will be used for outbreak detection algorithms to minimize the expected squared error of the forecast. Outbreak detection algorithms include Cumulative Sums (CUSUM), SMART, scan statistics, recursive least squares (RLS), wavelet-detection algorithms, and What is Strange About Recent Event (WSARE). Several of these algorithms will be used in order to compare the results. First, a combination of CUSUM and EWMA will predict next-day counts and monitor the differences between the cumulated average and predictions. The second monitoring tool will be a recursive least squares (RLS) algorithm, which fits "an autoregressive model to the counts and updates estimates continuously by minimizing prediction error" (8). The third tool will include a wavelet approach, which "decomposes the time series using Haar wavelets, and uses the lowest resolution to remove longterm trends from the raw series" (8). The residuals are then monitored using an ordinary 


\section{Development and Implementation of a Surveillance Network System for}

Emerging Infectious Diseases in the Caribbean (ARICABA)

Shewhart I-chart with a threshold of 4 standard deviations (8). By comparing these algorithms, it will monitor whether all the monitoring tools predict similar patterns, rather than relying on one forecasting algorithm.

In developing a forecasting algorithm, data clustering will be performed to organize the pattern of data, based on similarity. Afterward, regression analysis will be conducted to find a relationship (coefficients) between the data. PASW (SPSS Modeler 13) will be used for the analysis. The pattern of data and associated factors will be plugged into a mathematical algorithm that incorporates environmental characteristics such as weather conditions and mosquito distribution to develop an outbreak forecasting algorithm for dengue fever. For the mathematical algorithm, Holt Winter's Multiplicative Seasonality model will be tested in the pilot.

\section{Discussion}

Benefit of the ARICABA surveillance system and design

One of the main benefits of the ARICABA surveillance system is that it will improve responsiveness and representativeness of the existing health systems in the three countries through an automated approach of the collection and processing of data and transmission of information from various sources. By doing so, the network system will homogenize and organize the data that are collected from relevant stakeholders in Dominica, St. Lucia and Martinique, utilizing information and communication technology. By incorporating a disease monitoring system for confirmed cases and a syndromic surveillance system for forecasting, it will empower public health professional to prepare and intervene effectively when outbreaks occur.

\section{Detection Algorithm}

In a syndromic surveillance system, one of the keys for accurate outbreak detection is well designed and tested mathematical algorithms. Although the ARICABA syndromic surveillance system uses the basic idea of the RODS's mathematical model, it is important to develop its algorithms from feedback in order to capture and predict the outbreak in the Caribbean. It is crucial to continuously question what other algorithm combinations are available and investigate lesson learned from other syndromic surveillance systems.

\section{Data Collection}

In order for the data warehouse to function properly, a strategic plan for collecting data is critical. Necessary data for the data analysis must be collected in sufficient amounts. In addition, data collection should occur in a timely and complete manner while following agreed upon data standards. Hospitals, labs, and clinics have different data systems in each country. When each DCP collects data from the data sources, each DCP must be able to convert the data into data warehouse system. However, the absence of medical informatics and electronic medical record systems in Martinique and Dominica remains a challenge.

\section{System Requirements}

System requirement must take the volume and size of data into account. It must consider the data such as the list of syndromes, weather conditions, and migration to be collected from the three islands. In addition, it is important to design system requirements in a flexible manner so that the 


\section{Development and Implementation of a Surveillance Network System for}

Emerging Infectious Diseases in the Caribbean (ARICABA)

system can expand in case of increased data volume in the future. The system requirement for the data warehouse should also consider the performance capacity of CPU and RAM for calculating the outbreak detection algorithms.

Sensitivity vs. Specificity

A critical component regarding the algorithms for outbreak detection is balancing the level of specificity. For example, if the system has specificity set to low, there will be a lot of "falsepositive" epidemic alerts, which will tire the system. On the other hand, if it is too specific, the system may miss true epidemics, which does not meet the purpose of the system either (2).

\section{Technology Gaps}

Since the level of economic development in all three countries is different, it is important to consider the technology gaps among the islands. Not only internet capacity, but more importantly, the gap in medical informatics is an immense challenge to implement automated data collection from hospitals and laboratory. The automated, real-time chief complaint collection from emergency departments or clinics is especially crucial for a syndromic surveillance system; implementing adequate medical informatics system in hospitals among the countries must be completed first in order for the syndromic system to remain fully functional. Another way to overcome the technology gap is to utilize a "component-oriented" development approach, which develops each component of system separately so that it can be a "plug-in" to other systems when needed.

\section{Languages}

As mentioned, the three countries speak different languages. Among a mixed language setting, it is important to establish multiple language systems for the entire surveillance system. For example, all data should be displayed in French, English and Creole. Also, data translation features are necessary to communicate between the three countries. For instance, data or information entered in English in St. Lucia should be able to be read as French in Martinique; this highly interactive language feature remains a challenge.

\section{Limitations}

This study involved several limitations. A holistic, contextual view of this project was not analyzed; only certain aspects of the parts of the project have been analyzed. Therefore, causal conclusions were difficult to obtain. For example, time in the fellowship was a limiting factor in accessing data; budget and cost-effective analyses could have been valuable quantitative information but were not completed. Additionally, a possibility of observer and interviewer bias in handling the qualitative data exists. There may be interviewer bias in handling the data because a majority of technical concepts originate from Martinique. Observer bias in collected data is reduced due to three fellows working on the project with different backgrounds and expertise. In addition, the areas in which fellows worked vary with some overlap. However, the information from different perspectives enriches the perspective and qualitative analysis of this study. Finally, a lack of technical documentation limited this study to rely mainly on interviews and discussion.

\section{Conclusion}

The ARICABA surveillance project was initiated to protect Caribbean citizens and tourists from 
emerging infectious diseases by using network systems for early warning and forecasting. The aim is to implement a comprehensive surveillance system that enhances health systems by connecting current structures and maximizes its capacity through information technology in the Caribbean, where resources are limited.

This paper recommends a system architecture and database system for data warehousing. It proposes two systems: a disease monitoring system and a syndromic surveillance system within the data warehouse to predict the outbreak and monitor/control the disease. Data contents, sources, collection and visualizations strategies have also been suggested. However, collecting data, especially chief complaints from ERs in real-time, remains a challenge where medical informatics system are limited. Also, full language support for the entire system is complex.

Considering these challenges, recommendations include reducing technology gaps, in terms of medical informatics, between three countries; improving real-time data collection for the syndromic surveillance system; and establishing multi-language support system. For development strategies, "component-oriented" development approaches are recommended for the sustainability of ARICABA. The Caribbean region should continue to move forward in their efforts to improve surveillance systems and protect the health of their citizens.

\section{Acknowledgements}

We would like to acknowledge CICOMSCA and the Regional Council Martinique for their support of this project. Additionally, many thanks to the Center for Global Health at the George Washington University for the opportunity to participate with ARICABA through their Global Health Service Fellowship.

\section{References}

[1] Bliss K. Health in Latin America and the Caribbean: Challenges and Opportunities for U.S Engagement, Center for Strategic and International Studies Global Health Policy Center. 2009; Washington, DC.

[2] Bucher B. ARICABA: An Emerging Infectious Disease Surveillance System, Implementation \& Evaluation Plan. 2010; Martinique.

[3] Chen H. AI for Global Disease Surveillance, IEEE Computer Society. 2009: 1- 18.

[4] Central Intelligence Agency (CIA). The World Factbook [Internet]; [cited October 15, 2011 ]. Available from: https://www.cia.gov/library/publications/the-world-factbook/

[5] Dieye M. Cancer Incidence in Martinique: a model of epidemiological transition. European Journal of Cancer Prevention 2007Apr; 16(2):95-101.

[6] Ducharme C. Understanding Implementation Barriers to an Electronic Emerging Infectious Disease Surveillance System in the Caribbean, ARICABA: A Case Study. 2011; the Department of Global Health, the George Washington University, Washington D.C.

[7] Heymann D, Rodier G. Global Surveillance of Communicable Diseases. Emerging Infectious Diseases. 1998; 4(3):362-65. 
[8] Shmueli G, Burkom S. Statistical Challenges Facing Early Outbreak Detection in Biosurveillance. Technometrics (Special Issue on Anomaly Detection). 2010; 52(1): $39-51$

[9] Teutsch S. Principles and Practices of Public Health Surveillance, 2nd. ed. New York: Oxford University Press; 2000. 406p.

[10] Zeng D. Syndromic Surveillance Systems: Public Health and Biodefense. Review of Information Science and Technology (ARIST) [Internet]. 2008 [cited 2011 July 15]; 42. Available from: http://iasec.eller.arizona.edu/docs/Zeng-Survey-manuscript-revised.pdf [11] Zeng D. Infectious Disease Informatics And Outbreak Detection. Medical Informatics. New York: Springer Science; 2005. 647p. 